\title{
Type I cryoglobulinemia related to Sjögren's syndrome and MGUS: a case report
}

\author{
Kamil Klimas, Rafał Małecki \\ Department of Angiology, Systemic Hypertension and Diabetology, Wrocław Medical University, Poland
}

\begin{abstract}
Cryoglobulinemia is a rare disease caused by the specific antibodies which precipitate at low temperatures being present within the blood serum. It is obsenved in the course of disorders such as autoimmune diseases, lymphoproliferative neoplasms, and infectious diseases - mainly HCV infections. Three types of cryoglobulinemia have been identified, differing in the type of immunoglobulins involved and symptoms being manifested. We are presenting a case of a 6 I-year-old female patient with type I cryoglobulinemia related to Sjögren's syndrome and complicated by monoclonal gammopathy of undetermined significance.
\end{abstract}

Key words: cryoglobulinemia, monoclonal gammopathy of uncertain significance (MGUS), Sjögren's syndrome (SS)

Acta Angiol 2020; 26, 2: 8I-84

\section{Introduction}

Cryoglobulinemia is a rare disease (I case per 100,000 individuals) [1], consisting of antibodies which precipitate at low temperatures (i.e. temperatures of below $37^{\circ} \mathrm{C}$ ) being present within the blood serum [2]. It is observed in the course of various disorders such as lymphoproliferative neoplasms, autoimmune diseases, and viral infections, with HCV being the main agent responsible for the latter [2]. Three types of cryoglobulinemia have been identified, differing in the type of immunoglobulins involved, the most common being referred to as type II. We are presenting a case of a $6 \mathrm{I}$-year-old female patient with monoclonal cryoglobulinemia (of type I) related to Sjögren's syndrome and complicated by monoclonal gammopathy of undetermined significance (MGUS).

\section{Case report}

A 6I-year-old female patient with a 4-year history of Sjögren's syndrome was admitted to the Department of Angiology, Systemic Hypertension, and Diabetology with calf ulcers which had appeared several years before presentation. Upon admission, the patient was in severe overall condition with the disease affecting both calves and involving extensive and strongly adherent necrotic lesions reaching down to the fascial and muscular levels and requiring administration of high-dose opioid analgesics (Figs I, 2). Laboratory investigations revealed features of activated inflammatory response (CRP 43 $\mathrm{mg} / \mathrm{l}$ [reference range $0-5 \mathrm{mg} / \mathrm{l}$, leukocytosis 12.14 $\mathrm{G} / \mathrm{l}$, normocytic anemia) and reduced levels of complement $\mathrm{C} 4[<0.08 \mathrm{~g} / \mathrm{l}$, reference range $0.1-0.4 \mathrm{~g} / \mathrm{l}]$ with unremarkable levels of complement $C 3[1.46 \mathrm{~g} / \mathrm{l}$, reference level $0.9-1.8 \mathrm{~g} / \mathrm{l}]$. High levels of rheumatoid factor $[323 \mathrm{IU} / \mathrm{ml}]$ and presence of anti-Ro (SS-A) and anti-La (SS-B) antibodies at the titer of I:3200 were also demonstrated. No deviations from reference ranges were identified in urinalysis results. $\mathrm{HBV}$ and $\mathrm{HCV}$ screening tests were negative.

The scope of diagnostic laboratory investigations was broadened due to the unclear origin of severe necrotic lesions. Refrigeration test revealed the presence of cryoglobulins, later to be confirmed in serum immunofixation assays (monoclonal lgM kappa immu- 


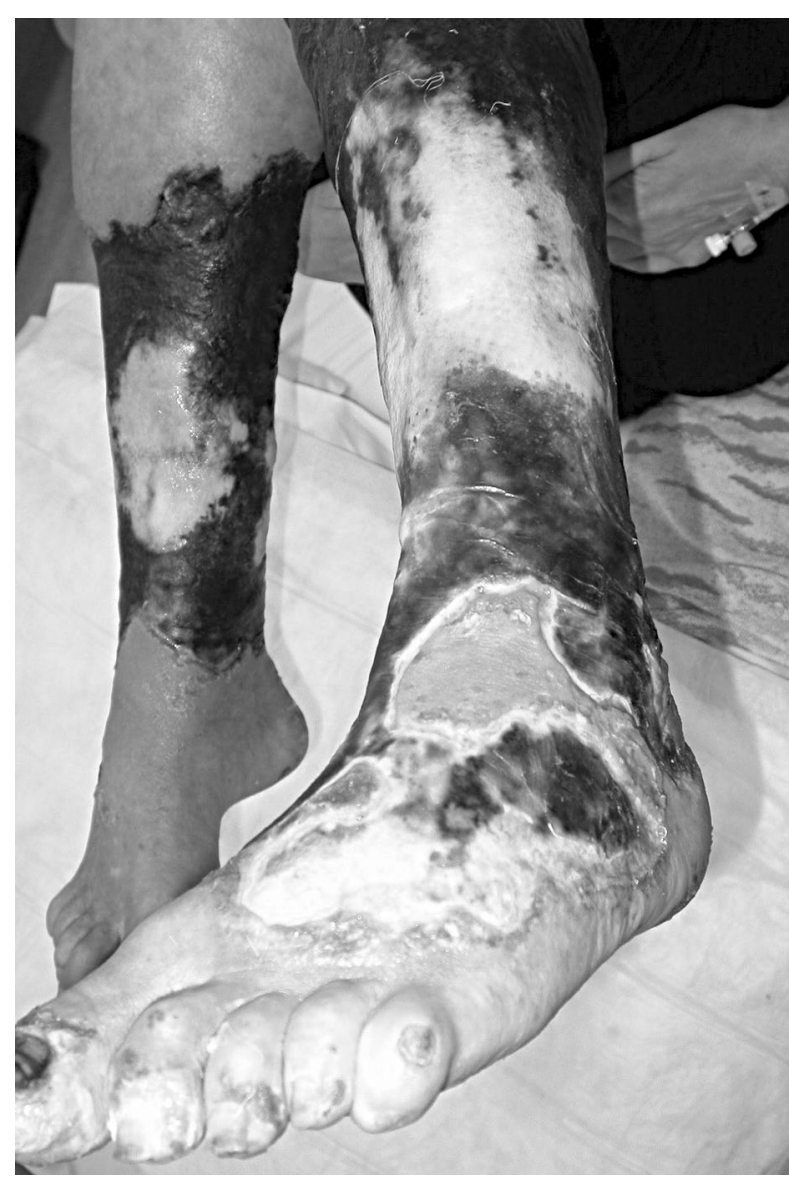

Figure I. Lesions before admission to the hospital

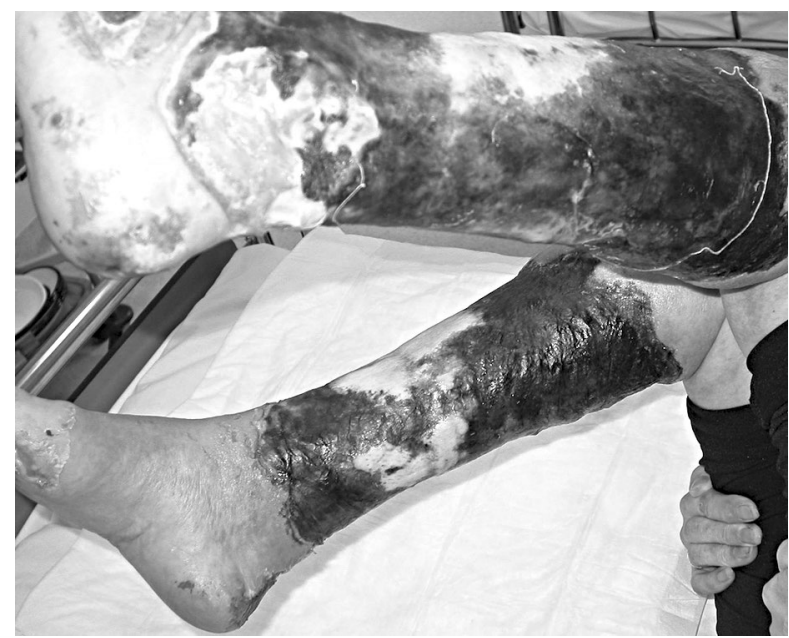

Figure 2. Lesions before admission to the hospital

noglobulin). Despite numerous additional assays (such as pANCA, cANCA, anti-dsDNA titers, HCV and HBV screening assays) symptoms of vasculitis related to other system dysfunctions were excluded. The patient had been subjected to hematological consultation which revealed monoclonal gammopathy of undetermined significance (MGUS). On the basis of clinical presentation and the results of laboratory investigations, the patient was diagnosed with vasculitis in the course of type I cryoglobulinemia secondary to MGUS which had developed as a complication of previously diagnosed Sjögren's syndrome.

Due to the aggressive course of the disease and the risk of resulting limb loss, a decision to initiate immunosuppressive therapy was made following hematological consultation. Between May 2014 and October 2016, a total of $7.6 \mathrm{~g}$ of cyclophosphamide was administered in pulses as an addition to local management (including mechanical debridement of ulcers under general anesthesia) to achieve partial healing of lesions. In October 2014 , the patient was diagnosed with asymptomatic proximal deep vein thrombosis and vertebral fractures at levels L2 and L4 (treated by vertebroplasty at the Department of Neurosurgery); after several days, another two fractures at $\mathrm{Th} / 2$ and $\mathrm{LI}$ were detected (and also subjected to surgical treatment). During subsequent hospitalizations, several episodes of airway infections were observed as was pulmonary and tricuspid regurgitation and dilatation of the aortic bulb.

Establishment of an efficient maintenance immunosuppression regimen was a significant problem. As the attempts to introduce methotrexate and azathioprine had failed (several airway infections), cyclosporine was identified as the best-tolerated drug. Currently, the ulcers are nearly completely healed (Figs 3,4), and the patient is capable of ambulating using a Zimmer frame. Laboratory investigations continue to show elevated CRP levels ( $12 \mathrm{mg} / \mathrm{I})$, microcytic anemia, and reduced GFR $\left(78 \mathrm{ml} / \mathrm{min} / 1.73 \mathrm{~m}^{2}\right)$. Cyclosporine is found to be present at optimum (therapeutic) levels.

\section{Discussion}

Sjögren's syndrome is a connective tissue disorder characterized by particularly profound and dangerous immunological disorders. Observations include increased proliferation of $B$ cells, and, as a consequence, production of numerous autoantibodies (anti-Ro/SSA, anti-La/SSB, AMA, anti-centromere antibodies) and rheumatoid factor as well as increased expression of pro-inflammatory cytokines, i.e. IL-I $\beta$, IL-6, IL- I 7, TNF- $\alpha$, interferon $\gamma$ [3]. As a result of numerous immunological mechanisms, the risk of non-Hodgkin lymphoma (NHL) is 4 to 44 times higher in patients with Sjögren's syndrome as compared to the overall population [4]. Monoclonal antibodies were detected in $7-22 \%$ of patients $[5,6]$; in some cases, the synthesized gamma-globulins presented with cryoglobulin features. 


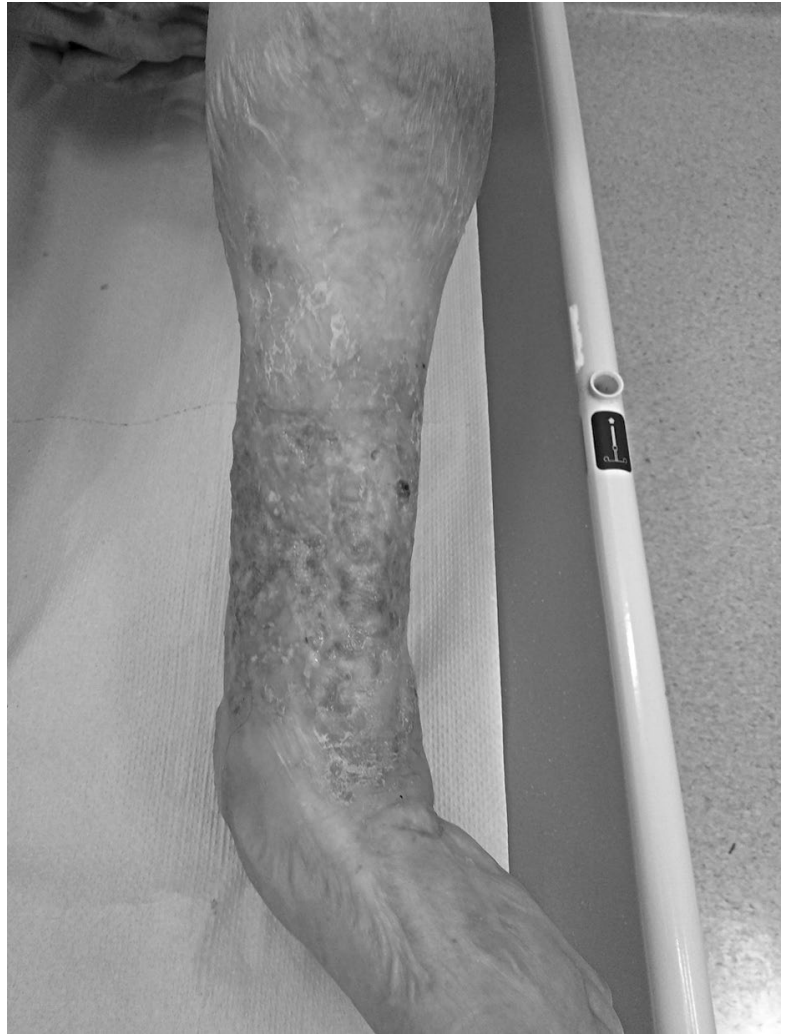

Figure 3. The left calf after treatment

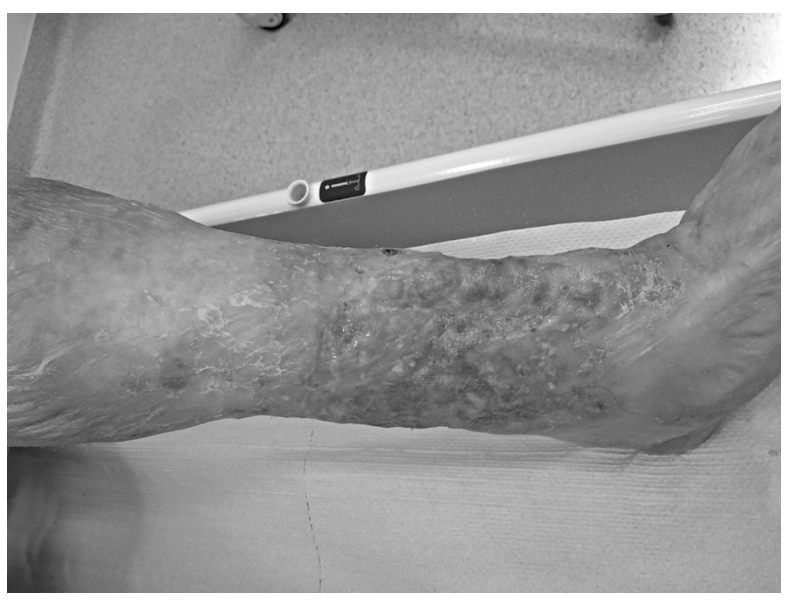

Figure 4. The left calf after treatment

Three types of cryoglobulinemia are identified on the basis of laboratory investigations: monoclonal (type I) cryoglobulinemia, mixed monoclonal/polyclonal (type II) cryoglobulinemia and polyclonal (type III) cryoglobulinemia. Each type may be manifested by a different set of symptoms. Type I cryoglobulinemia is characterized mainly by the presence of skin lesions such as purple-colored papulae on lower limbs, livedo reticularis, and Raynaud's phenomenon as well as the most serious lesions including ulcers and tissue necrosis which were predominant in the clinical presentation of the reported patient. Types II and III more frequently present with symptoms within other systems, with strong muscle and joint pains becoming more intensive at lower temperatures, peripheral polyneuropathy, hepatic dysfunction, respiratory symptoms (cough, dyspnea, pleuritis) or membranoproliferative glomerulitis. These symptoms are associated with cryoglobulins being deposited within small vessels (mainly capillaries, venules, or arterioles) and present in the blood.

Symptoms observed in the reported case, i.e. skin lesions including calf ulcers and soft tissue necrosis correspond to type I cryoglobulinemia. Other disorders taken into consideration in differential diagnosis included i.a. Schnitzler syndrome (absence of typical urticaria, recurrent fever, lymphadenopathy, or joint pains), secondary vasculitis related to Sjögren's syndrome and Waldenström macroglobulinemia (absence of recurrent epistaxis).

No standard of treatment has been established to date for cryoglobulinemia. The treatment must target the diseases involved in the development of the disorder. Cryoglobulinemia-causing neoplasms (chronic lymphocytic leukemia, Waldenström macroglobulinemia, and other types of lymphoma) are treated with chemotherapy, sometimes with adjuvant radiotherapy. Cryoglobulinemia in the course of HCV or HBV infection (mixed type II or type III cryoglobulinemia) is treated with antiviral regimens (the authors' experience suggests that eradication of the hepatitis $C$ virus leads to symptom resolution). Patients with symptomatic cryoglobulinemia and vasculitis syndromes, as in the presented case, are treated with immunosuppressants.

Cyclophosphamide (an alkylating agent) was shown to efficiently induce remission in the presented case; however, the choice of remission maintenance treatment proved to be problematic. Glucocorticosteroids cause resolution of symptoms while simultaneously reducing cryocrit values. Cytotoxic drugs such as azathioprine (a purine antimetabolite) and methotrexate (a folic acid antagonist) ) facilitate inhibition of immunoglobulin production by B cells. In the reported patient, however, this treatment proved ineffective due to disease complications. Cyclosporine was identified as the most effective and well-tolerated treatment. Cyclosporine belongs to the group of calcineurin inhibitors and inhibits humoral and cellular immune responses modifies chronic inflammatory processes, reduces the production and secretion of lymphokines (such as IL-2) and T-cell growth factor (TCGF) as well as affects the activity of T helper cells. In addition, cyclosporine inhibits the induction phase of the lymphatic cell proliferation process. 
We are presenting this case due to the extraordinary intensity of necrotic lesions resulting from the presence of monoclonal proteins with cryoglobulin properties rather than from secondary vasculitis. It appears that every case of unexplained skin lesions or necrotic lesions in patients suffering from Sjögren's syndrome or, in a broader sense, an autoimmune disease, requires that this disease entity is taken into consideration in differential diagnostics.

\section{Conflict of interest}

None.

\section{References:}

I. Takada S, Shimizu T, Hadano Y, et al. Cryoglobulinemia (review). Mol Med Rep. 2012; 6(I): 3-8, doi: 10.3892/mmr.2012.86I, indexed in Pubmed: 22484457.
2. Muchtar E, Magen H, Gertz MA. How I treat cryoglobulinemia. Blood. 2017; 129(3): 289-298, doi: 10.1182/ blood-2016-09-7/9773, indexed in Pubmed: 27799164.

3. Huang Yf, Cheng $\mathrm{Q}$, Jiang $\mathrm{Cm}$, et al. The immune factors involved in the pathogenesis, diagnosis, and treatment of Sjogren's syndrome. Clin Dev Immunol. 2013; 2013: 160491, doi: 10.1 I55/2013/16049I, indexed in Pubmed: 23935646.

4. Pillemer SR. Lymphoma and other malignancies in primary Sjögren's syndrome. Ann Rheum Dis. 2006; 65(6): 704-706, doi: 10.1 I36/ard.2005.044362, indexed in Pubmed: 16699050.

5. Tomi AL, Belkhir R, Nocturne G, et al. Brief report: monoclonal gammopathy and risk of lymphoma and multiple myeloma in patients with primary Sjögren's syndrome. Arthritis Rheumatol. 2016; 68(5): 1245-1250, doi: 10.1002/art.39534, indexed in Pubmed: 26636425.

6. Brito-Zerón P, Retamozo S, Gandía M, et al. Monoclonal gammopathy related to Sjögren syndrome: a key marker of disease prognosis and outcomes. J Autoimmun. 20I2; 39(I-2): 43-48, doi: 10.1016/j.jaut.2012.01.010, indexed in Pubmed: $22297 \mid 46$ 